\title{
CCSSM: A TOOLKIT FOR INFORMATION EXTRACTION FROM REMOTELY SENSED IMAGERY
}

\author{
Y. Ge ${ }^{\mathrm{a}, *}$, C. Zhang ${ }^{\mathrm{a}, \mathrm{b}}, \mathrm{H}$. X. Bai ${ }^{\mathrm{a}}$ \\ ${ }^{a}$ State Key Laboratory of Resources and Environmental Information System, Institute of Geographic Sciences \& \\ Natural Resources Research, Chinese Academy of Sciences, Beijing - (gey, zhangch, baihx)@lreis.ac.cn \\ ${ }^{\mathrm{b}}$ School of the Earth Sciences and Resources of China University of Mine and Technology, Xuzhou
}

KEY WORDS: Information Extraction, Spatial Information, Spectral Information, Multiple-point Simulation

\begin{abstract}
:
This paper presents a method named CCSSM (Classification of Combining Spectral information and Spatial information upon Multiple-point statistics) which is the derivation of two probability fields from the supervised classification for the spectral extraction and multiple-point simulation (MPS) for the spatial information, which then are fused. The performance of CCSSM for two-class classification has been discussed in our previous research works. This paper mainly introduces the software toolkit of CCSSM. A multiple-class classification using CCSSM is then given.
\end{abstract}

\section{INTRODUCTION}

Incorporating spatial structural information and spatial correlation information with spectral information is one way to improve the accuracy of classification of remotely sensed imagery (Ge et al., 2008b). In past decades, much effort has been directed towards developing excellent methods such as contextual classification, classification using texture structural information, and classification utilizing geostatistics. Recently, Ge et al. (2006; 2008a; 2008b; 2009) introduced multiplepoint simulation (MPS) into the process of information extraction to increase the classification accuracy of remotely sensed imagery. MPS was used to characterize the structural and spatial association properties of geographical objects through a training image. MPS is one of the main applications of multiple-point statistics (Zhang et al. 2005), while SNESIM is one of the effective algorithms of MPS. The method of integrating MPS with spectra information was named as the Classification of Combining Spectral information and Spatial information upon Multiple-point simulation (CCSSM) (Ge et al., 2008c). The performance of CCSSM for the two-class classification has been substantiated by experiment and accuracy assessment using a Landsat Thematic Mapper (TM) $30 \mathrm{~m}$ image (Ge et al., 2008a; Ge et al., 2008b). This paper mainly introduces the software toolkit of CCSSM. A multiple-class classification using CCSSM is then given.

\section{CCSSM}

\subsection{General idea}

CCSSM is the derivation of two probability fields from the supervised classification and multiple-point simulation (MPS) and based on spectral and spatial information, which then are fused. CCSSM completely uses the training image and the template information to acquire the structural information of geographical objects and compensates for the inadequacy of using only spectral information for the information extraction.
It not only takes spectral information of the remotely sensed imager into account, but also considers the spatial structure information and spatial correlation information of geographical objects. Therefore, it can effectively extract the investigated object with distinct spatial structural characteristics through a training image, and in particular can assist the user to extract objects smaller than the spatial resolution of the imagery (Ge et al., 2008a ,2008b and 2009).

In previous studies, the experiment undertaken (Ge et al., 2008a; 2008b and 2009) was only concerned with two-class classification, for instance, road and non-road. In fact, the algorithm is not limited to two-class classification. For example, assume that there are three categories with distinct structure characteristics. There are two means to simulate there classes. (1) First draws three training images which correspond to the simulations of three categories. Second, arbitrarily select a category as the first category to be simulated with the corresponding training image and all other categories will be treated as one category. Then the second category is simulated versus all other categories pooled together except the first category. Similarly, the third category can be simulated. (2) The second means is to first draw one training image with three categories and then simulate three categories simultaneously with the training image. In this paper, the second means will be implemented in the example.

\subsection{CCSSM toolkit}

This toolkit of CCSSM is written by $\mathrm{c}^{++}$with GTK. This toolkit is composed of three parts for processing input data, intermediate data and output data as shown in Figure 1. The input data includes the probability field from the supervised classification which could be maximum likelihood classification (MLC) and training image. The supervised classification can be carried out in commercial software package for remotely sensed data such as PCI Geomatica, EARDAS and IDRISI. The training image can be drawn in commercial image software such as Photoshop, and then converted into a raster text file as input data.

\footnotetext{
* Corresponding author. gey@lreis.ac.cn.
} 
The second part is for processing the intermediate data which includes hard data and search tree. The hard data for MPS can be obtained from the training data for MLC or the classified results from MLC. The search tree is a dynamic data structure that stores the conditional probability distribution of the training image and allows retrieval of all conditional probability distributions existing in a training image (Strebelle 2000, Ge et al., 2008b). Single normal equation simulation (SNESIM) is one of the effective algorithms of MPS. In CCSSM, SNESIM is adopted to extract the spatial structural information (Strebelle 2000). In the SNESIM algorithm, the template is an important parameter in the simulation process. It is a search window that consists of a set of ranked pixels and MPS use it to capture patterns from the training image. The SNESIM algorithm with multiple grid approach is performed to simulate the unsampled data through training image and then obtain the MPS result (Ge et al., 2009).

The third part is for fusing the probability fields from MPS and MLC. CCSSM toolkit provides three kinds of fusion methods which are the consensus-based fusion method, the evidencebased fusion method and the probability-based fusion method. In CCSSM toolkit, the formats of input data, intermediate data, and output data are the text type.

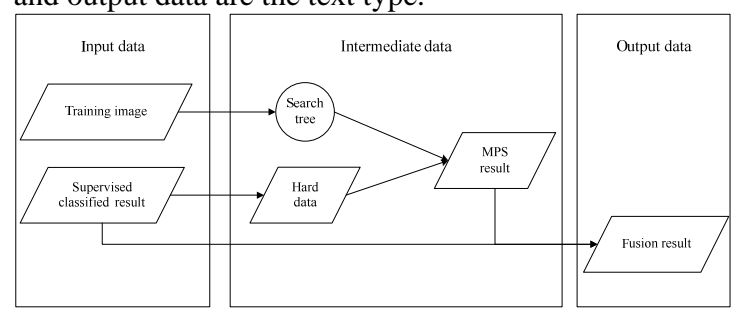

Figure 1. Components and data flow in CCSSM toolkit

\section{EXAMPLE}

The experimental procedure consists of four steps: MLC classification, MPS, data fusion using the consensus theory, and an accuracy assessment using error matrices. As described before, we first perform the MLC classification, then obtain MPS results with the revised SNESIM algorithm, and finally fuse the results using the consensus theory.

\subsection{Data description}

The example data was selected from QUICKBIRD $2.5 \mathrm{~m}$ imagery. The image size is $614 \times 787$ pixels and its resolution is $2.5 \mathrm{~m}$ as shown in Figure 2. From this image, it can be seen the roads and buildings have strong spatial structural features; for instance, in the study area, most roads are of a cross network structure and a certain inclination and most buildings distribute in square in the middle of the image.

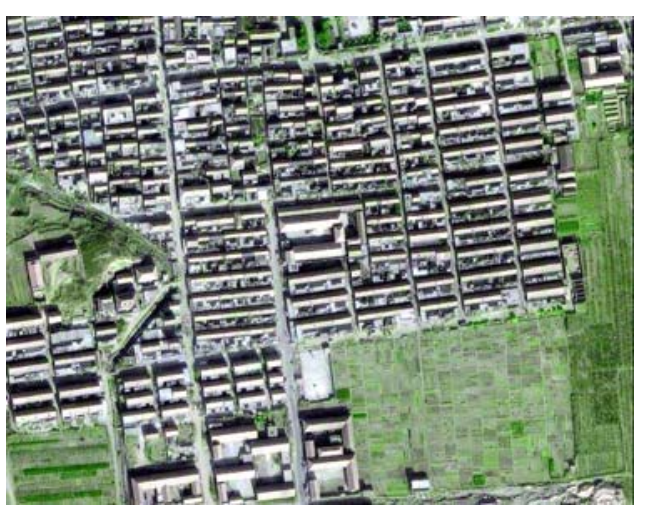

Figure 2. QUICKBIRD $2.5 \mathrm{~m}$ imagery

\subsection{Training image}

The training image was drawn by hand according to common characteristics of interested objects. In this remotely sensed imagery, the classes of buildings, gardens, roof shadow and streets have obvious structures and their spatial distributions are also homogeneous except in those areas of lawn.

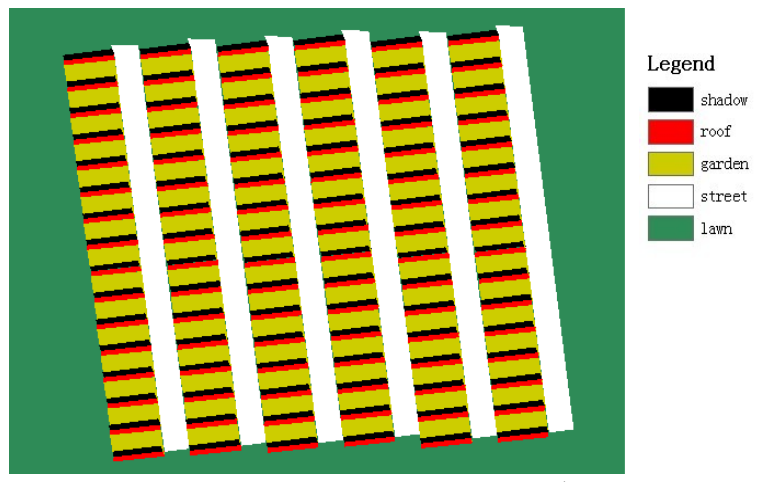

Figure 3. Training image (560x400)

\subsection{MLC}

From the Figure 2, the geographical objects can be roughly classified into 5 classes which are building roof, garden between the buildings, street road, lawn and roof shadow. As to investigate the performance of CCSSM method on multipleclass classification, the training data is selected in according the five classes. Then the MLC classification algorithm is performed using PCI Geomatica.

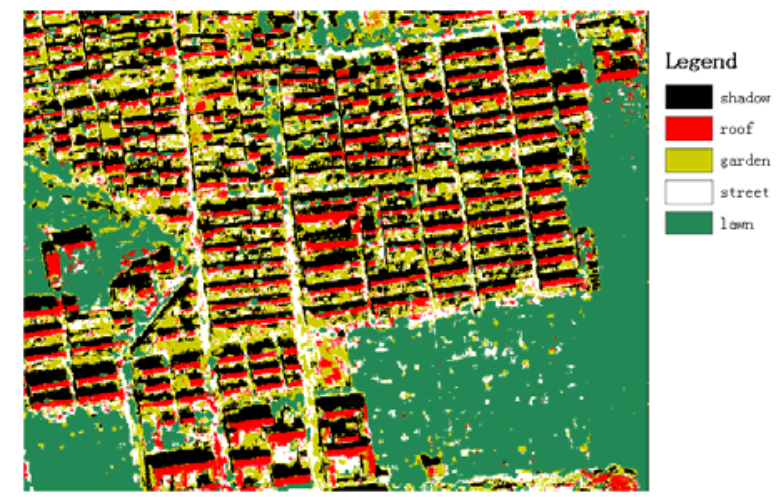

Figure 4. MLC results 


\subsection{MPS simulation}

3.4.1 Hard data: There are two ways to obtain the hard data for MPS as shown in Figure 5. One is to use directly the sample data for MLC as hard data. The other is to select some pixels from the classified results of MLC by setting a threshold such as 0.7 . The threshold can be set in terms of the resolution of remotely sensed imagery and the classification accuracy of MLC.

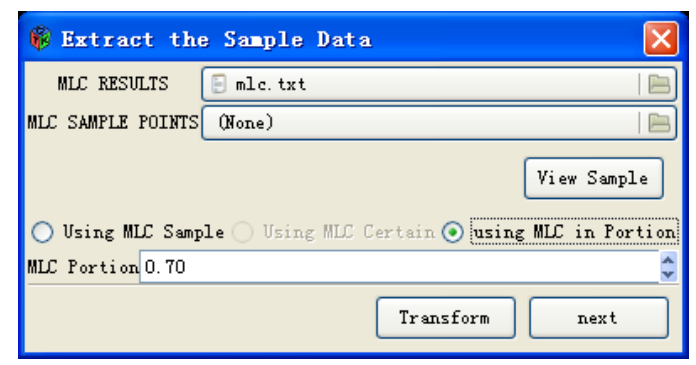

Figure 5. Interface for extracting sample data for MPS

3.4.2 SNESIM: First select the training image and press "View Train-Image" to view the training image as shown in Figure 6. The function of the button of "Make Template" is to set the template, for instance, the template can be set $5 \times 5$ or7 7 pixels. The grid size is to set the parameter of multiple-grid approach. This parameter can be set to $1,2,4,8, \ldots$ Sort Interval denotes to sort the random path again after simulating a fixed number of pixels (Ge et al., 2008b). After setting all parameters, press the button of SNESIM and then get MPS result as shown Figure 7.

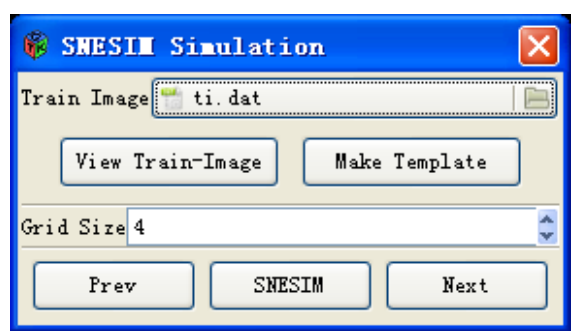

Figure 6. Interface for setting parameters for SNESIM

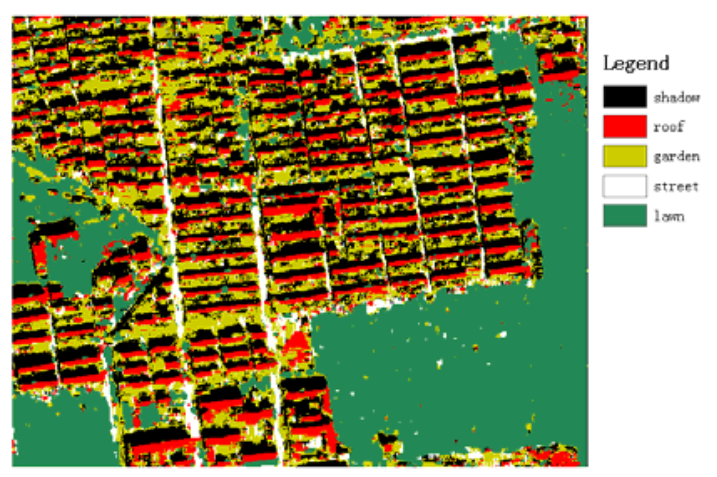

Figure 7. MPS result

\subsection{Fusion}

First one presses the button of "Transform" to extract the probability filed from MLC and then selects the fusion method as shown in Figure 8. In this example, the CONSENSUS-based fusion method is chosen to fuse two classification results. Finally, one presses the button of "Data Fusing" to obtain the fusion result as shown Figure 9. The result then is saved to the format of text file and can be viewed in ENVI software package.

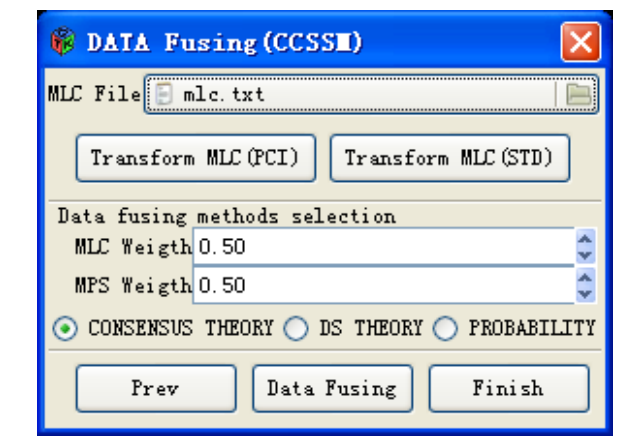

Figure 8. Interface for setting parameters for Fusion

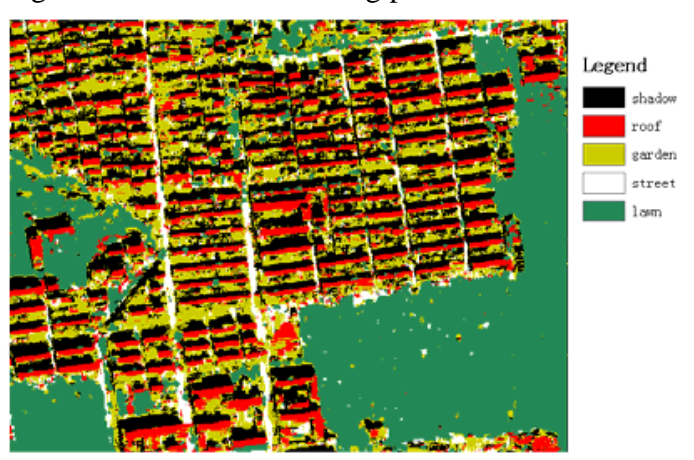

Figure 9. Fusion result

\section{CONCLUSION AND FUTURE WORKS}

This paper briefly introduced the general idea of CCSSM and its toolkit. A multiple-class classification is then given to demonstrate the use of CCSSM. Experiments have shown that this method can effectively extract the interested feature information with distinct structural characteristics. Furthermore, there are some aspects need to be discussed. For example, the software toolkit needs further improvement and can be downloaded for free to interested users around the world. The template is designed by a more reasonable and scientific way which considers the spatial structure and scale characteristics of the study area. The training image is a key factor in MPS and directly influences the simulation results, therefore, it is essential to design the training image with a realistic design.

\section{REFERENCES}

Ge, Y., Bai, H.X. and Le, D.Y., 2006, A classification method for remotely sensed imagery by integrating with spatial structure information. In 7th International Symposium on Spatial Accuracy Assessment in Natural Resources and Environmental Sciences. 5-7 JULY 2006, Lisboa, Portugal (Lisboa: Instituto Geográfico Português), pp. 588-594.

Ge, Y., Bai, H.X., Cheng, Q.M., 2008a, The solution of multiple-point statistics to extracting information from remotely sensed imagery. Journal of Chinese University of Geoscience, 19, pp. 421-428.

Ge, Y., Bai, H.X., Cheng, Q.M., 2008b, New classification method for remotely sensed imagery via multiple-point 
simulation: experiment and assessment. Journal of Applied Remote Sensing, 2, 023537.

Ge, Y., Bai, H.X., 20009, Multiple-point simulation-based method for extraction of objects with spatial structure from remotely sensed imagery. International Journal of Remote Sensing (accepted).

Strebelle, S. (2000). Sequential simulation drawing structures from training images: Unpublished doctoral dissertation, Stanford University, California.

Zhang, T., Switzer, P. and Journel, A., 2005, Merging prior structural interpretation and local data: The bayes updating of multiple-point statistics, In Proceeding of International Association for Mathematical Geology, 21-26 August 2005, Toronto, Canada (Toronto: the Geomatics Research Laboratory, York University and Wuhan: China University of Geosciences), pp. 615-620.

\section{ACKNOWLEDGMENTS}

This work was supported in part by the National High Technology Research and Development Program of China (Grant No 2006AA06Z115, 2006AA120106) and the National Natural Science Foundation of China (Grant No. 40671136, 40971222) and State Key Laboratory of Independent Innovation Team Project (Grant No. O88RA203SA). 